\title{
Imagined rotations of self versus objects: an fMRI study
}

\author{
Maryjane Wraga $^{\mathrm{a}, *}$, Jennifer M. Shephard ${ }^{\mathrm{b}}$, Jessica A. Church ${ }^{\mathrm{a}}$, \\ Souheil Inatic ${ }^{\mathrm{c}}$, Stephen M. Kosslyn ${ }^{\mathrm{b}}$ \\ ${ }^{a}$ Department of Psychology, Smith College, Science Center, Bass Hall 304, Northampton, MA 01063, USA \\ ${ }^{\mathrm{b}}$ Department of Psychology, Harvard University, Cambridge, MA 02138, USA \\ ${ }^{\mathrm{c}}$ Center for Neural Science and Department of Psychology, New York University, New York, NY, USA
}

Received 9 January 2003; received in revised form 23 September 2004; accepted 16 November 2004

Available online 20 January 2005

\begin{abstract}
This study used functional magnetic resonance imaging (fMRI) to investigate the neural mechanisms underlying two types of spatial transformations: imagined object rotations and imagined rotations of the self about an object. Participants viewed depictions of single threedimensional Shepard-Metzler objects situated within a sphere. A T-shaped prompt appeared outside of the sphere at different locations across trials. In the object rotation task, participants imagined rotating the object so that one of its ends was aligned with the prompt. They then judged whether a textured portion of the object would be visible in its new orientation. In the self rotation task, they imagined rotating themselves to the location of the T-prompt, and then judged whether a textured portion of the object would be visible from the new viewpoint. Activation in both tasks was compared to respective control conditions in which identical judgments were made without rotation. A direct comparison of self and object rotation tasks revealed activation spreading from left premotor to left primary motor (M1) cortex (areas 6/4) for imagined object rotations, but not imagined self rotations. In contrast, the self rotation task activated left supplementary motor area (SMA; area 6). In both transformations, activation also occurred in other regions. These findings provide evidence for multiple spatial-transformation mechanisms within the human cognitive system.
\end{abstract}

(C) 2004 Elsevier Ltd. All rights reserved.

Keywords: Mental imagery; Mental rotation; Motor learning; fMRI

\section{Introduction}

If a person wants to know what an object looks like from a different viewpoint without actually moving, there are at least two mental transformations she can try. She can imagine rotating the object until the desired viewpoint is aligned with her current perspective, or she can imagine moving herself around the object to the new viewpoint. Both of these mental transformations are important in everyday tasks of spatial reasoning. Each requires the representation of a different spatial reference frame. Imagined object rotations involve transformation of the object-relative reference frame, which specifies the location of an object's parts with respect to each other (Easton \& Sholl, 1995). Imagined self rotations involve

\footnotetext{
* Corresponding author. Tel.: +1 413585 3988; fax: +1 4135853786

E-mail address: mwraga@smith.edu (M. Wraga).
}

transformation of the egocentric reference frame, which specifies an object's location and orientation with respect to the intrinsic axes of the observer's body (Howard, 1982). The egocentric frame also can be specified at smaller scales to relate objects to specific parts of the body, such as the head or hand.

An important question is whether the mental transformations associated with object-relative and egocentric reference frames are subserved by different neural mechanisms. If different neural systems are activated during the two types of transformation, this is solid evidence that different mechanisms are in play. In recent years, researchers have used neuroimaging techniques to explore this issue. Most studies have examined imagined object rotations (e.g., Barnes et al., 2000; Carpenter, Just, Keller, Eddy, \& Thulborn, 1999; Cohen et al., 1996; Kosslyn, DiGirolamo, Thompson, \& Alpert, 1998; Lamm, Windischberger, Leodolter, Moser, \& Bauer, 2001; 
Richter et al., 2000; Tagaris et al., 1997; Vingerhoets et al., 2001). Some have examined imagined rotations of bodies (e.g., Creem et al., 2001a). The few studies directly comparing both classes of mental rotation have yielded ambiguous evidence for distinct mechanisms (Zacks, Ollinger, Sheridan, \& Tversky, 2002; Zacks, Rypma, Gabrieli, Tversky, \& Glover, 1999; Zacks, Vettel, \& Michelon, 2003). In the present study, we used functional magnetic resonance imaging (fMRI) to identify the neural substrates underlying imagined self and object rotations, using novel rotation tasks.

The first hints that different spatial transformations may be subserved by different mechanisms were evident in behavioral studies that compared how easily participants can imagine rotating an array versus how easily they can imagine rotating themselves around the array (e.g., Amorim \& Stucchi, 1997; Creem, Wraga, \& Proffitt, 2001b; Huttenlocher \& Presson, 1997; Presson, 1982; Wraga, Creem, \& Proffitt 2000; Wraga, Creem-Regehr, \& Proffitt, 2004). In these experiments, participants typically perform one or the other type of imagined rotation and then update the location of a given object in an array. Researchers consistently have found faster and more accurate performance during imagined self rotations than during imagined rotations of the array. Moreover, the response time (RT) functions corresponding to each type of imagined rotation show unique characteristics. RTs for imagined array and object rotations tend to increase linearly when greater amounts of rotation are required, which suggests that observers mentally transform objects similarly to the way objects are physically transformed (Shepard \& Metzler, 1971). In contrast, RTs for imagined self rotations usually are independent of rotation magnitude beyond $0^{\circ}$, with the exception of angles that are oblique to the intrinsic axes of the body (Wraga, 2003; Wraga et al., 2000, 2004). Performance also is unaffected by physically impossible situations, such as imagining rotating one's body around an array that is parallel to a wall (Creem et al., 2001b). Thus, although they conform to some physical laws constraining the body, imagined self rotations generally exhibit more flexibility than their physical counterparts.

Neuroimaging studies have begun to elucidate the neural correlates of imagined self and object rotations. For mental rotation of objects, it is well established that the posterior parietal lobules play a major role. Many studies have found bilateral posterior parietal activation, with the greatest concentration in the left superior parietal lobule (area 7) (e.g., Kosslyn et al., 1998; Richter et al., 2000; Tagaris et al., 1997; Vingerhoets et al., 2001). Recent studies have begun to delimit the precise function of the superior parietal lobule in mental rotation (e.g., Harris \& Miniussi, 2003; Podzebenko, Egan, \& Watson, 2002). For example, Podzebenko et al. (2002) used fMRI to demonstrate that changes in cerebral blood flow within the superior parietal lobule are positively correlated with the magnitude of rotation of a mentally rotated object. This finding suggests that the superior parietal lobule is intimately involved in the process of altering the representation of an object's orientation per se, perhaps by mapping transformations of the object-relative reference frame.

Some neuroimaging studies also have provided evidence that low-level motor areas (specifically, premotor and primary motor [M1] areas) are activated during mental rotation. Investigators initially reported motor activation for mental transformations of body-related stimuli such as hands and feet. They interpreted these findings as evidence that participants had imagined rotating their own body parts to solve the tasks (e.g., Bonda, Petrides, Frey, \& Evans, 1995; Kosslyn et al., 1998; Parsons et al., 1995). However, a growing number of studies have reported motor activation during mental rotation of nonbody objects (e.g., Bonda et al., 1995; Carpenter et al., 1999; Cohen et al., 1996; Kosslyn, Thompson, Wraga, \& Alpert, 2001; Richter et al., 2000; Tagaris et al., 1997; Vingerhoets et al., 2001). For example, Cohen et al. (1996) examined mental rotation of the Shepard and Metzler (1971) figures with fMRI and found premotor activation in half of their participants. Using positron emission tomography (PET), Kosslyn et al. (2001) examined whether participants could voluntarily adopt motor strategies during mental rotation. Kosslyn et al. found that the primary motor area M1 was activated when participants were instructed to solve a mental rotation task by imagining objects being turned by their hands; in contrast, no such activation occurred when participants imagined the objects being rotated by an external source, such as an electric motor. Such activation of motor areas also has been shown to transfer implicitly from an imagined hand rotation task to an object rotation task (Wraga, Thompson, Alpert, \& Kosslyn, 2003). Richter et al. (2000) provided evidence that motor activation is not epiphenomenal to the mental rotation process, but rather plays an integral part. They found that peak activation in motor areas correlated positively with participants' RTs in a mental rotation task. These findings suggest that low-level motor activation plays a role in the process of mentally rotating nonbody objects, perhaps by transforming spatial signals from the posterior parietal lobule into movement signals (see also Ganis, Keenan, Kosslyn, \& Pascual-Leone, 2000; Harris \& Miniussi, 2003; Snyder, Batista, \& Andersen, 2000; Wexler, Kosslyn, \& Berthoz, 1998).

The mechanisms underlying imagined self rotations are less well understood. Creem et al. (2001a) used fMRI to examine performance in an imagined self rotation task. Participants memorized the locations of four objects in an array, and then updated the objects' locations after performing imagined "log-roll" transformations of their bodies about the array's center. Similar to mental rotation of objects, Creem et al. found bilateral superior parietal activation with stronger activation in the left cerebral hemisphere. They also reported left premotor area (PMA; area 6) and supplementary motor area (SMA; area 6) activation, but no M1 activation. Zacks et al. (2002) attempted to directly compare imagined object and self rotations using fMRI. In their study, participants viewed stimuli of human bodies and performed two mental rotation tasks. In one, participants made same-different 
judgments of a pair of bodies misoriented with respect to each other, essentially treating the bodies as objects to be rotated into congruence. In the other, participants judged whether one of two bodies had a right or left arm extended, a task requiring an egocentric transformation. Both tasks elicited typical areas of activation found in mental rotation tasks, including bilateral activation in superior parietal lobules and in premotor areas. However, a direct comparison yielded no distinctive cortical regions across the two tasks, only different relative amounts of activity. In general, Zacks et al. (2002) found greater cortical activation in the same-different task versus the right-left task within the right posterior cortex.

One explanation for Zacks et al.'s (2002) failure to identify distinct brain areas that underlie the different classes of rotation is that their tasks were ambiguous. It is possible that participants construed the left-right task as an imagined hand rotation task rather than an imagined self rotation task. That is, participants may have performed the task by simply imagining rotating their hands into the stimuli, rather than imagining the perspective change required of imagined self rotations. In the present study, we designed tasks that require explicit rotations in order to identify the neural activation underlying imagined self and object rotations. Participants viewed depictions of a single object situated within a sphere and made judgments about its appearance after either imagining rotating the object or imagining rotating themselves around the object. Based on the results of previous mental rotation studies, we expected to find distinctive mechanisms for imagined object and self rotations. Specifically, we predicted that posterior parietal activation would occur in both rotation tasks, but that low-level motor area activation would be restricted to imagined object rotations.

\section{Method}

\subsection{Participants}

We recruited 11 right-handed individuals ( 4 females, 7 males; mean age: 25 years; range: $20-38$ years) from the Dartmouth College community. The data from one additional participant were excluded because of anatomical anomalies. Handedness was determined with the Edinburgh handedness scale (Oldfield, 1971). Prior to the study, all participants gave written consent to the protocol as approved by Harvard University and Dartmouth College. Participants were paid US\$ 20 for their participation.

\subsection{Materials}

The stimuli were depictions of the three-dimensional, multi-armed cube figures originally used by Shepard and Metzler (1971), rendered with Bryce 3D software (Metacreations Corp., New York, NY). Each object was depicted within a sphere. One of the inner cubes of each object was textured. For the object-rotation task, a three-dimensional Tshaped prompt appeared on the end of one arm of the object; a second T-prompt appeared somewhere outside of the sphere (see Fig. 1a). For the self rotation task, the T-prompt appeared only on the outside of the sphere (see Fig. 1b). For each rotation task, we used two different objects, which were rotated in increments of $65^{\circ}, 100^{\circ}$, and $135^{\circ}$ in either the $X$ (frontal) or $Y$ (transverse) planes of rotation for a total of 12 stimuli. From the 12 self stimuli and 12 object stimuli, we created two orders of trials for each rotation task. For the control task, similar objects appeared within spheres, but no T-prompts were present.
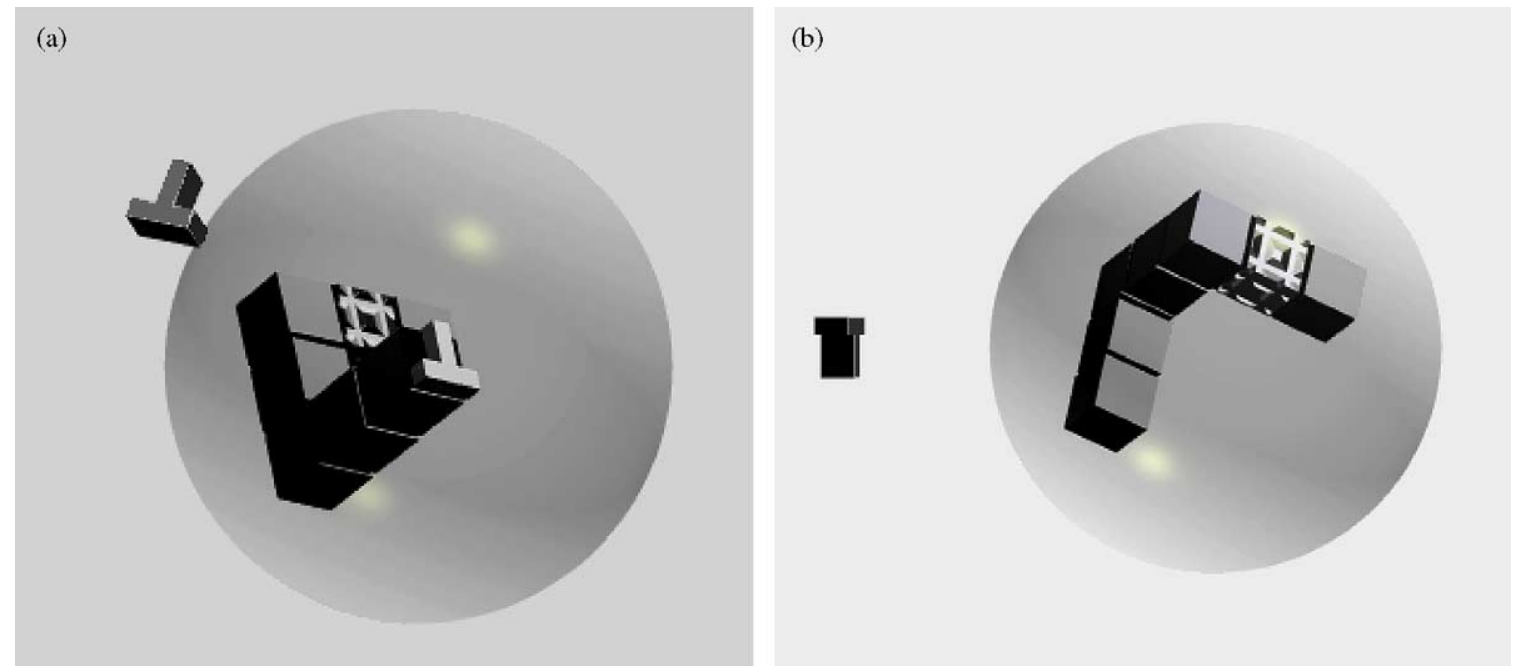

Fig. 1. The stimuli used in the experiment. (a) In the object rotation task, participants were asked to imagine rotating the object until the white T on its near end became aligned with the T-prompt outside of the sphere. They then judged whether the object's textured cube was visible given the object's new orientation. The correct answer for this trial is "yes." (b) In the self rotation task, participants were asked to imagine rotating themselves to the location of the T-prompt. They then judged whether the object's textured cube was visible from that new perspective. The correct answer for this trial is "no". 
Stimuli were displayed on a Macintosh PowerBook G3 computer using PsyScope software (Cohen, MacWhinney, Flatt, \& Provost, 1993), which also recorded responses and RTs. During fMRI scanning, the stimuli were back-projected onto a screen positioned $1.6 \mathrm{~m}$ from the participant. The projected objects (including the spheres and T-prompts) were $40 \mathrm{~cm}$ in diameter. As viewed by the participant in the scanner, this corresponded to approximately $2.5^{\circ}$ of horizontal visual angle. During the pre-scan training session, stimuli were displayed on the computer monitor.

\subsection{Tasks}

\subsubsection{Self rotation}

Participants were asked to imagine rotating their bodies about the sphere until their eyes lined up behind the horizontal line of the T-prompt, as if they were looking at the object through the T. They then made a "yes" or "no" decision as to whether the interior textured cube would be visible from that new perspective.

\subsubsection{Object rotation}

Participants were asked to imagine rotating the object so that the T-prompt attached to it lined up with the T-prompt outside the sphere. They then made a "yes" or "no" decision as to whether the interior textured cube would be visible (from their current perspective) given the object's new orientation.

\subsubsection{Self-control and object control}

Participants viewed the objects and made a "yes" or "no" decision as to whether the interior textured cube was visible. No rotation was necessary to make this judgment.

\subsection{Procedure and design}

Participants first were trained on the tasks outside the scanner, in the same order they performed them in the scanner. After reading the instructions for each task, participants performed six practice trials with feedback, one trial at each rotation and axis of rotation, using stimuli that did not appear in the test trials.

Each set of test trials began with a fixation point that remained on the screen for $30 \mathrm{~s}$, followed by four alternating blocks of the control and rotation tasks, for a total duration of $268 \mathrm{~s}$. Control stimuli were presented for $2800 \mathrm{~ms}$, with a $500 \mathrm{~ms}$ inter-stimulus interval, in blocks of nine trials. Rotation stimuli were presented for $9500 \mathrm{~ms}$, with a $500 \mathrm{~ms}$ inter-stimulus interval, in blocks of three trials. All stimuli remained on the computer screen for the durations stated, irrespective of the amount of time the participant required to respond. Self and object rotation trials were presented in separate sets of trials.

Participants performed two sets of trials for each rotation task. They responded in the scanner by pressing one of two buttons on a button box with their dominant hands. The buttons were covered with different textures to help participants distinguish them by touch, and were connected to the Macintosh computer via the PsyScope button box. After participants completed the first rotation task, they paused to review the instructions for the next rotation task, and scanning began again after the investigator was satisfied that they understood the task.

The order of rotation task (self versus object) was counterbalanced across participants. Trials in each set were presented in a pseudo-random order with the following restrictions: The same response could not occur three times in succession, and the same rotation magnitude could not be repeated until all variations had appeared once. Order of trials within each set of rotations (e.g., Self 1, Self 2) was kept constant across participants.

\section{5. fMRI acquisition}

Imaging was performed on a $1.5 \mathrm{~T}$ GE Signa CV/NVi LX8.3 MRI scanner (GE Medical Systems, Wakesha, WI). We obtained four functional sets of trials (134 scans each) in a single session for each participant. Four additional scans at the beginning of each set were discarded to ensure steadystate conditions. A standard head coil with foam padding for head stabilization was used. Functional images were acquired with a single-shot gradient echo EPI sequence, with parameters $\mathrm{TR}=2000 \mathrm{~ms}, \mathrm{TE}=35 \mathrm{~ms}$, flip angle $=90^{\circ}, 27$ contiguous $5 \mathrm{~mm}$ thick axial slices with an in-plane resolution of $64 \times 64$ in a FOV of $240 \mathrm{~mm}$. T1-weighted structural images were acquired at the same slice locations to aid in registration $(\mathrm{TR}=650 \mathrm{~ms}, \mathrm{TE}=6.6 \mathrm{~ms})$. Immediately following the functional scans, high-resolution, 3D T1-weighted structural images were acquired.

\subsection{Imaging analysis}

The data were analyzed using Statistical Parametric Mapping (SPM99) (Friston et al., 1995). Motion artifacts were corrected to the first functional scan for each participant. The 27-slice structural image was then co-registered to the high-resolution structural image, and the parameters of the resulting transformation were applied to the mean of the motion-corrected images as well as to all motion-corrected functional images. The functional images were then directly co-registered to the high-resolution structural image via mutual-information co-registration. The images were spatially normalized to the Montréal Neurological Institute template (which averages over 152 brains) and then smoothed with a Gaussian filter of $6 \mathrm{~mm}$ full-width half maximum (FWHM) to compensate for anatomical differences among participants.

We analyzed images using a two-stage, random effects analysis. First, we conducted within-subject, whole-brain analyses using a fixed-effects model under assumptions of the General Linear Model. The three tasks were modeled as box-car functions, convolved with a standard hemodynamic response function (Friston et al., 1995). We then performed a 
second, group-level analysis for each contrast, in which subject was treated as a random effect. The group-level analysis was based on one-sample $t$-tests thresholded at $p<.005$ (uncorrected for multiple comparisons) with an extent threshold of five contiguous voxels. This corresponds to a 0.0013 false positive rate for each statistical parametric map (Forman et al., 1995). The resulting clusters of activation were converted from MNI to Talairach-Tournoux space (Brett, 2002).

\section{Results}

\subsection{Behavioral results}

\subsubsection{Response times}

Fig. 2a shows mean RTs and standard errors for both rotation tasks as a function of rotation magnitude. The principal finding was that participants were faster at updating in the self task $(M=3.737 \mathrm{~s})$ than in the object task $(M=4.641 \mathrm{~s})$. A 2 (task order) $\times 2$ (rotation task) $\times 3$ (rotation magnitude) mixed design analysis of variance (ANOVA) performed on mean scores yielded a main effect of task $F(1,9)=10.19, p<.011$. Although the effect of rotation magnitude was not significant $(p=.229)$, we found a significant Condition $\times$ Rotation Magnitude interaction, $F(2$, $18)=11.19, p<.001$. Linear contrasts performed for each rotation task yielded the following patterns. For the self task, RTs decreased from $65^{\circ}$ to $100^{\circ}(t(10)=-2.89, p<.016)$, and increased from $100^{\circ}$ to $135^{\circ}(t(10)=2.84, p<.017)$. For the object task, RTs increased from $65^{\circ}$ to $100^{\circ}(t(10)=3.77$, $p=.004)$, and remained constant between $100^{\circ}$ and $135^{\circ}$ $(t(10)=0.31, p<.765)$.

For the $0^{\circ}$ control condition, which was identical in object and self rotation tasks, we collapsed the data across tasks (see Fig. 2a). As would be expected, participants were much faster in these control trials $(M=1.259 \mathrm{~s} ;$ S.E. $= \pm 126.13)$.

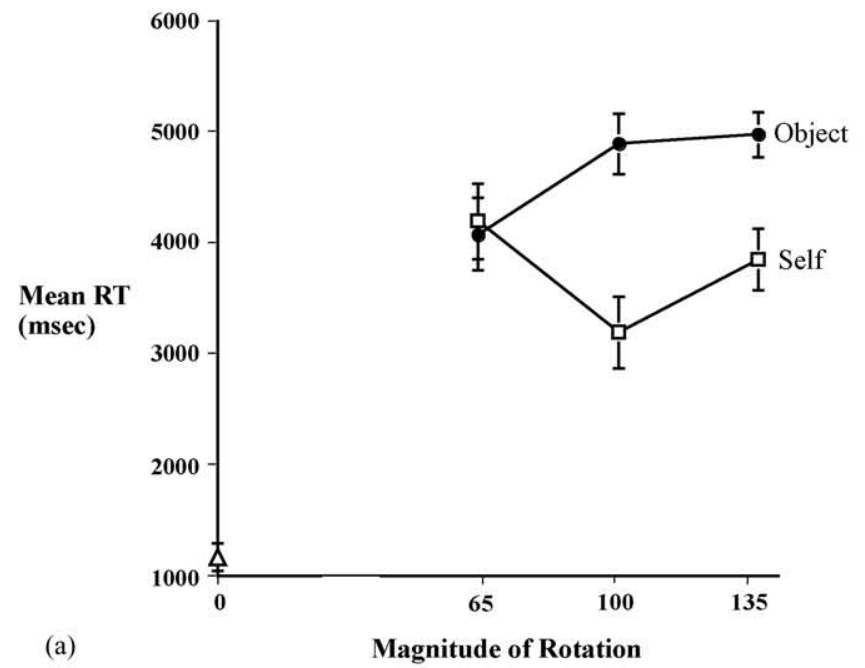

\subsubsection{Accuracy}

Fig. $2 \mathrm{~b}$ shows mean proportion error and standard errors for each rotation task as a function of angular disparity. Participants' responses were slightly but significantly more accurate in the self task $(M=10 \%$ error) than in the object task $(M=13 \%$ error). A 2 (task order) $\times 2$ (rotation task) $\times 3$ (rotation magnitude) mixed design ANOVA performed on mean error scores produced main effects of task, $F(1,9)=5.69, p<.041$, and rotation magnitude, $F(2$, $18)=6.00, p<.01$, and a significant Task $\times$ Rotation Magnitude interaction, $F(2,18)=11.65, p<.001$. Linear contrasts performed for each rotation task yielded the following patterns. For the self task, errors decreased from $65^{\circ}$ to $100^{\circ}(t(10)=6.71, p<.0001)$, and increased from $100^{\circ}$ to $135^{\circ}(t(10)=-2.81, p<.018)$. For the object task, errors remained constant between both comparisons $\left(65^{\circ}-100^{\circ}: t(10)=0.42, p=.683 ; 100^{\circ}-135^{\circ}: t(10)=1.70\right.$, $p=.120)$.

For the $0^{\circ}$ control condition, we again collapsed the data across tasks (see Fig. 2b). Participants were highly accurate in responding to these control trials $(M=1 \%$ error; S.E. $= \pm 0.003)$.

\section{2. fMRI results}

The purpose of this study was to examine the neural substrates underlying imagined self versus object rotations. To achieve this, we first analyzed activations and deactivations for the two rotation tasks with respect to the control task. We then directly compared patterns of activation in the self and object rotation tasks. In addition to providing basic comparisons, the analyses relative to the control task allowed us to assess whether the different patterns of activation we found when directly comparing object and self conditions were the result of decreases, rather than increases, within regions. Thus, for each significant area of activation found

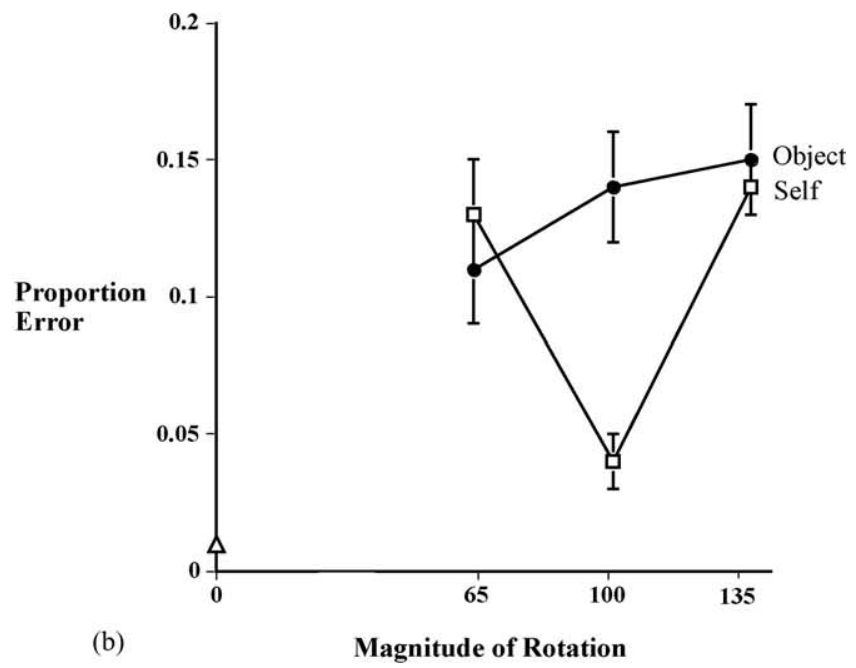

Fig. 2. (a) Mean response times and (b) mean proportion error for trials in the object and self rotation tasks, as a function of rotation magnitude. Data from the $0^{\circ}$ condition are collapsed over object and self rotation tasks. 
within the object-self and self-object contrasts, we computed $t$-statistics with respect to the object-control and self-control contrasts.

Tables 1 and 2 present the results of the comparisons between rotation tasks and control task. In general, the results for the imagined object rotation task are similar to those reported by previous researchers investigating mental rotation of objects (e.g., Cohen et al., 1996; Kosslyn et al., 1998). The comparison of the object task to the control task revealed left premotor area (area 6) extending to M1. We also found bilateral activation in the superior parietal lobule (area 7), a region typically associated with spatial transformations of objects, as well as primary visual cortex (area 17). Regarding deactivations, the object-control contrast revealed several clusters, including the left paracentral lobule (area 5), left superior frontal gyrus (area 8), right lateral sulcus (areas 22/39), and right DLPFC (area 9). These results are consistent with those of previous studies designed to assess decreases in activation across a range of visual cognition tasks (e.g., Shulman et al., 1997).

In contrast, the comparison of the self task to the control task revealed no activation in low-level motor areas. We found left activation in pre-SMA (area 6) and right activation in the superior parietal lobule (area 7). The findings generally are in line with Creem et al.'s (2001a) study of imagined self rotations. Additional areas of activation included the anterior cingulate (area 32), the DLPFC (areas 9/10), the inferior temporal gyrus (area 37), and bilateral fusiform gyrus (area 20). For self-control deactivations, we found several regions including left occipital areas (area 18), right superior temporal gyrus (area 22), left inferior frontal gyrus (area 45), and left superior frontal gyrus (area 8).
As shown in Table 3 and Figs. 3 and 4, when we directly compared the results from the two rotation tasks, we found several different areas of activation between them. For the object-self contrast, we again found activation in the left PMA, extending into M1 (see Fig. 3). We found activation in the left superior parietal lobule (area 7) and also in inferior parietal lobule (area 40) bilaterally. Activation also was evident in the portion of the dorsolateral prefrontal cortex (DLPFC) involved in spatial working memory (area 9), as well as in associative visual areas (areas 18/19), although the $t$-contrast performed for the latter region indicated that activation there was only marginally greater than that in the control task. In contrast, the reverse comparison of self-object revealed no PMA or M1 activation (see Fig. 4). We found activation in the left SMA (area 6), although this again was only marginally greater than in the control task. We also found activation in the left middle occipital gyrus at the junction with the fusiform gyrus (areas 19/37) and the right middle temporal gyrus (area 21), although the latter again was only marginally different from what occurred in the control task. Activation in the left insula and the right superior frontal gyrus (area 8) was found to be the result of relatively greater deactivation in the object task than in the self task.

\section{Discussion}

In this study, we used two novel mental rotation tasks to investigate the neural underpinnings of imagined self versus object rotations. Although we found that some common cortical regions were activated during the two types of transformations, for the most part they evoked several distinct areas

Table 1

Areas of activation and deactivation in the object rotation task with respect to the control

\begin{tabular}{|c|c|c|c|c|c|c|}
\hline Object task & Brodmann area(s) & $X$ & $Y$ & $Z$ & $t$-Value & Cluster size $\left(\mathrm{mm}^{3}\right)$ \\
\hline \multicolumn{7}{|l|}{ Activations } \\
\hline Superior parietal lobule & 7 & -12 & -60 & 51 & 14.35 & 2789 \\
\hline Superior parietal lobule & 7 & -4 & -59 & 55 & 13.89 & \\
\hline Superior parietal lobule & 7 & 24 & -63 & 51 & 12.73 & \\
\hline PMA/M1 & $6 / 4$ & -36 & 2 & 48 & 13.94 & 2384 \\
\hline Inferior temporal gyrus & 37 & -55 & -47 & -14 & 5.95 & 38 \\
\hline Cuneus & 17 & 8 & -69 & 11 & 5.47 & 12 \\
\hline Lingual gyrus & 17 & -4 & -93 & 1 & 3.53 & 5 \\
\hline \multicolumn{7}{|l|}{ Deactivations } \\
\hline Paracentral lobule & 5 & -8 & -25 & 49 & 12.63 & 1351 \\
\hline Transverse temporal gyrus & 41 & -40 & -19 & 5 & 12.03 & 487 \\
\hline Insula & & -48 & -7 & 11 & 7.40 & \\
\hline Cuneus & 19 & -20 & -92 & 27 & 7.39 & 140 \\
\hline Cuneus & 18 & -20 & -97 & 12 & 7.11 & \\
\hline DLPFC & 9 & 12 & 56 & 34 & 7.81 & 114 \\
\hline Superior frontal gyrus & 8 & -16 & 45 & 38 & 7.45 & 27 \\
\hline Superior frontal gyrus & 8 & -20 & 37 & 39 & 6.54 & \\
\hline Rectal gyrus & 11 & 4 & 34 & -22 & 7.40 & 146 \\
\hline Rectal gyrus & 11 & -8 & 38 & -12 & 6.15 & \\
\hline Superior temporal gyrus & 39 & 55 & -61 & 29 & 5.65 & 81 \\
\hline Lateral sulcus & $22 / 39$ & 60 & -53 & 25 & 5.08 & \\
\hline Lingual gyrus & 18 & 24 & -94 & -5 & 5.20 & 9 \\
\hline
\end{tabular}

Talairach and Tournoux (1988) coordinates for activation peaks and maxima $t$-values are provided. 
Table 2

Areas of activation and deactivation in the self rotation task with respect to the control

\begin{tabular}{|c|c|c|c|c|c|c|}
\hline Self task & Brodmann area(s) & $X$ & $Y$ & $Z$ & $t$-Value & Cluster size $\left(\mathrm{mm}^{3}\right)$ \\
\hline \multicolumn{7}{|l|}{ Activations } \\
\hline Pre-SMA & 6 & -32 & 10 & 44 & 14.40 & 448 \\
\hline DLPFC & 9 & -48 & 13 & 29 & 11.06 & \\
\hline Superior parietal lobule & 7 & 24 & -59 & 58 & 12.26 & 2490 \\
\hline Anterior cingulate & 32 & 4 & 32 & 28 & 10.24 & 159 \\
\hline Medial frontal gyrus & 8 & -4 & 29 & 35 & 9.42 & \\
\hline Junction of mid./ant. cingulate & $24 / 32$ & -8 & 36 & 17 & 6.33 & \\
\hline Inferior frontal gyrus & $11 / 47$ & 32 & 23 & -15 & 8.56 & 40 \\
\hline Inferior temporal gyrus & 37 & -52 & -51 & -11 & 5.95 & 10 \\
\hline DLPFC & 10 & 20 & 62 & -6 & 4.83 & 6 \\
\hline Fusiform gyrus & 20 & 40 & -32 & -22 & 4.15 & 9 \\
\hline Fusiform gyrus & 20 & -52 & -32 & -19 & 3.88 & 5 \\
\hline \multicolumn{7}{|l|}{ Deactivations } \\
\hline Inferior occipital gyrus & 18 & -28 & -93 & -2 & 8.54 & 167 \\
\hline Cuneus & 18 & -12 & -97 & 12 & 6.31 & \\
\hline Superior temporal gyrus & 22 & 52 & 4 & 0 & 8.50 & 250 \\
\hline Postcentral gyrus & 3 & -55 & -13 & 45 & 4.71 & 91 \\
\hline SMA & 6 & -8 & -9 & 59 & 5.06 & 205 \\
\hline Parahippocampal gyrus & 34 & -16 & -13 & -16 & 6.85 & 104 \\
\hline Parahippocampal gyrus & 34 & -32 & -21 & -26 & 5.58 & \\
\hline Medial frontal gyrus & 10 & -4 & 50 & 3 & 6.49 & 230 \\
\hline Inferior frontal gyrus & 45 & -55 & -30 & 16 & 6.60 & 104 \\
\hline Superior temporal gyrus & 41 & -44 & -31 & 9 & 6.57 & \\
\hline Postcentral gyrus & 3 & 36 & -21 & 42 & 6.44 & 265 \\
\hline Postcentral gyrus & 3 & 44 & -17 & 52 & 6.42 & \\
\hline Postcentral gyrus & 3 & 36 & -17 & 49 & 6.40 & \\
\hline Middle temporal gyrus & 39 & 55 & -65 & 29 & 5.71 & 46 \\
\hline Superior temporal gyrus & 39 & -55 & -61 & 25 & 5.11 & 11 \\
\hline Superior temporal gyrus & 22 & -59 & -50 & 10 & 4.41 & 9 \\
\hline Parahippocampal gyrus & 36 & 24 & -17 & -26 & 3.75 & 18 \\
\hline Middle temporal gyrus & 21 & 59 & -9 & -16 & 4.01 & 6 \\
\hline Middle temporal gyrus & 21 & -59 & -4 & 4 & 3.81 & 9 \\
\hline Middle temporal gyrus & 21 & -55 & 0 & -7 & 3.53 & \\
\hline
\end{tabular}

Talairach and Tournoux (1988) coordinates for activation peaks and maxima $t$-values are provided.

Table 3

Areas of activation in the object rotation task compared to the self rotation task (top) and vice versa (bottom)

\begin{tabular}{|c|c|c|c|c|c|c|c|c|}
\hline & $\begin{array}{l}\text { Brodmann } \\
\operatorname{area}(\mathrm{s})\end{array}$ & $X$ & $Y$ & $Z$ & $t$-Value & $\begin{array}{l}\text { Cluster size } \\
\left(\mathrm{mm}^{3}\right)\end{array}$ & $\begin{array}{l}t \text {-Value } \\
\text { (object-control) }\end{array}$ & $\begin{array}{l}t \text {-Value } \\
\text { (self-control) }\end{array}$ \\
\hline \multicolumn{9}{|l|}{ Object-self } \\
\hline PMA/M1 & $6 / 4$ & -32 & -6 & 43 & 7.16 & 165 & 13.62 & 9.09 \\
\hline Inferior frontal gyrus & $44 / 47$ & -52 & 8 & 7 & 5.93 & 18 & 3.27 & -0.33 \\
\hline Inferior parietal lobule & 40 & 55 & -37 & 46 & 5.86 & 19 & 4.74 & 3.97 \\
\hline DLPFC & 9 & 40 & 44 & 31 & 5.78 & 45 & 3.89 & 0.27 \\
\hline Inferior parietal lobule & 40 & -44 & -33 & 39 & 5.08 & 38 & 7.94 & 7.33 \\
\hline Inferior frontal gyrus & 47 & 32 & 23 & -11 & 5.05 & 45 & & \\
\hline Cuneus & $18 / 19$ & 8 & -88 & 27 & 4.67 & 5 & 0.80 & -0.38 \\
\hline Inferior parietal lobule & 40 & 40 & -33 & 42 & 3.67 & 16 & 8.49 & 5.22 \\
\hline Superior parietal lobule & 7 & -28 & -48 & 54 & 3.60 & 9 & 5.25 & 4.92 \\
\hline \multicolumn{9}{|l|}{ Self-object } \\
\hline SMA & 6 & 0 & -24 & 56 & 7.15 & 62 & -1.69 & 0.78 \\
\hline Superior frontal gyrus & 8 & 20 & 30 & 46 & 5.01 & 23 & -3.26 & -0.36 \\
\hline $\begin{array}{l}\text { Middle occipital gyrus/fusiform } \\
\text { gyrus }\end{array}$ & $19 / 37$ & -55 & -73 & 4 & 4.36 & 13 & 1.42 & 3.04 \\
\hline Insula & & -44 & -8 & 8 & 4.16 & 5 & -6.27 & -2.43 \\
\hline Medial temporal gyrus & 21 & 60 & -35 & -5 & 4.13 & 15 & -2.50 & 0.47 \\
\hline
\end{tabular}

Talairach and Tournoux (1988) coordinates for activation peaks and maxima $t$-values are provided, as well as $t$-values for each major area of activation with respect to object-control and self-control contrasts. 


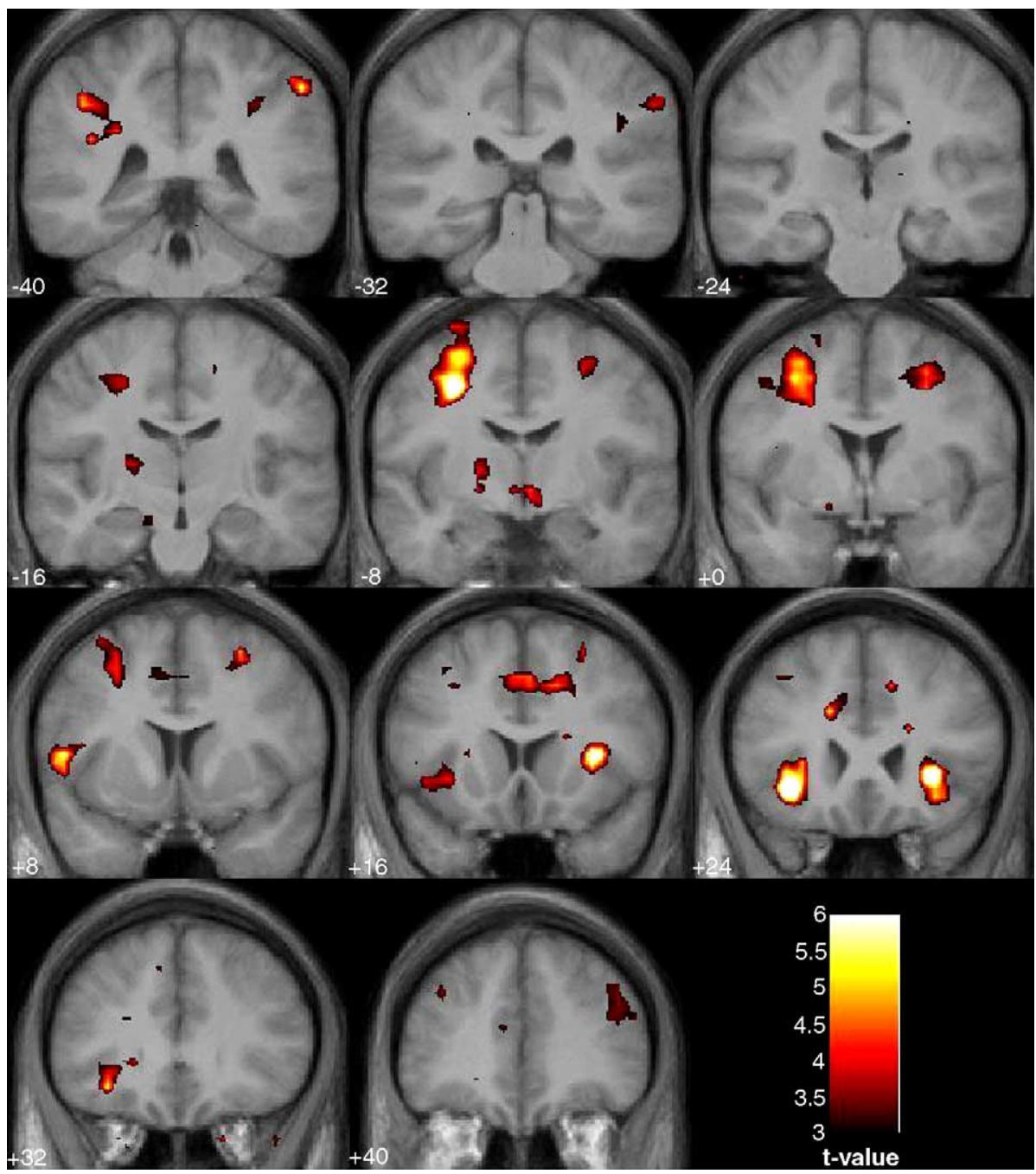

Fig. 3. Coronal images $(y=-40$ to +40$)$ depicting activation resulting from the object-self contrast. Areas depicted include PMA/M1/inferior frontal gyrus, and inferior and superior parietal lobules. Activation is superimposed onto a brain image created from the average of all participants.

of activation. As predicted, one clear-cut difference involved low-level motor activation, which we found in the object task but not the self task. Our analysis of deactivations indicated that this result could not be attributed to corresponding regions of deactivation in the individual object and self tasks. This dissociation between imagined self and object rotations is in line with a growing body of empirical evidence indicating that imagined spatial transformations are subserved by multiple neural mechanisms (e.g., Kosslyn et al., 2001; Wraga et al., 2003; Zacks et al., 1999).

We found evidence for distinct neural mechanisms when we compared each rotation task with the control task (see Tables 1 and 2). The object-control contrast revealed left activation in PMA extending to M1. We also found bilateral activation in the posterior parietal lobule (area 7). These findings are similar to those of other studies on mental rotation of objects (e.g., Cohen et al., 1996; Kosslyn et al., 1998; Richter et al., 2000; Tagaris et al., 1997; Vingerhoets et al., 2001). The self-control contrast revealed activation of the region of
SMA thought to underlie complex spatial judgments (Picard \& Strick, 1996) but no low-level motor activation. This contrast also yielded right activation in a spatial processing area (area 7). These findings are again in line with Creem et al.'s (2001a) study of imagined self rotations. However, one finding that conflicts with Creem et al. and other neuroimaging studies of mental rotation is the absence of activation in visual areas (areas 17, 18, 19). Instead, we found significant deactivations in area 18, which previously has been associated with the execution of eye movements (Paus, Marrett, Worsley, \& Evans, 1995). In general, however, analyses of individual conditions support the notion of two distinct mechanisms for imagined object and self rotations.

We also found support for distinct neural mechanisms for the two kinds of transformation in the direct comparison of imagined object and self rotation tasks (see Table 3). The analysis for the object-self contrast revealed activation in left premotor area (area 6) extending to area M1. These areas previously have been implicated in preparatory hand 


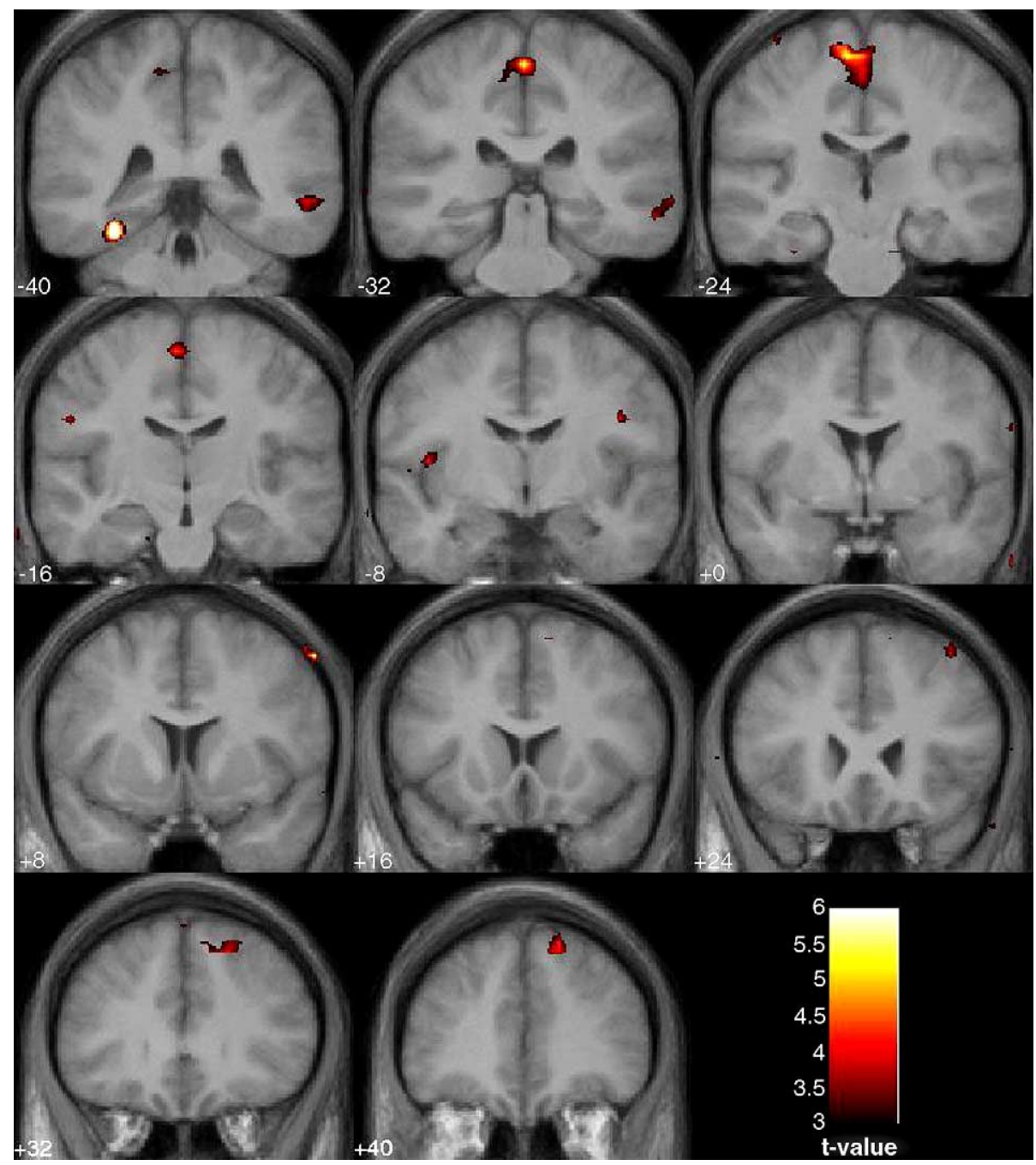

Fig. 4. Coronal images $(y=-40$ to +40$)$ depicting activation resulting from the self-object contrast. Areas depicted include SMA, middle occipital gyrus, and medial temporal gyrus. Activation is superimposed onto a brain image created from the average of all participants.

movements (e.g., He, Dumm, \& Strick, 1995; Rizzolati et al., 1998). Moreover, several mental rotation studies have reported similar regions of activation in tasks in which participants imagined rotating objects with their hands, either explicitly or implicitly (Kosslyn et al., 2001; Tagaris et al., 1997). It is likely that participants in the current study used a similar motor strategy for the imagined object rotation task. This possibility is bolstered by the fact that the M1 activation we found was limited to the left cerebral hemisphere, which controls the right hand (all of our participants were strongly right-handed).

In contrast, the reverse comparison of self-object revealed no PMA or M1 activation, but activation in the SMA, which lies very high in the "motor hierarchy" and has been found to be activated in many cognitive tasks (e.g., Crozier et al., 1999; Raichle, 1998). Although the magnitude of the SMA activation was not significantly greater than in the control condition, this may have been a consequence of the relatively high-level control task we used (cf. Zacks et al., 2003). In performing the control task, participants initially may have encoded each stimulus with respect to egocentric coordinates, which has been shown to be the default reference system for encoding spatial information (e.g., Shelton \& MacNamara, 2001). Such a strategy could account for the similar magnitude of activation for self and control tasks. This issue notwithstanding, the absence of motor activation for imagined self rotations is consistent with the findings of Creem et al. (2001a). Apparently, imagining oneself moving around an object does not involve imagining oneself walking or otherwise moving muscles.

The self-object comparison also revealed activation in left middle occipital gyrus at the junction of the fusiform gyrus, a result that is broadly consistent with other studies of egocentric tasks involving imagined movement (e.g., Zacks et al., 1999, 2003). For example, Zacks et al. (2003) found left activation in the PTO junction when directly comparing brain activation involved in imagined viewer-object rotations. Activation in the present study was located in a region associated with visual motion perception (e.g., Dupont, Orban, De 
Bruyn, Verbruggen, \& Mortelmans, 1994), which suggests a role for imagined movement as well.

In line with previous behavioral studies, participants were faster and more accurate at performing imagined self rotations than imagined object rotations (e.g., Wraga et al., 2000, 2004). It might be argued that the different patterns of brain activation we found across tasks were due to differences in difficulty, rather than differences between the two mental transformations per se. However, this interpretation seems unlikely for two reasons. First, the activation we found for imagined self and object rotations generally is consistent with fMRI studies that examined each class of rotation in isolation (e.g., Cohen et al., 1996; Creem et al., 2001a; Kosslyn et al., 1998; Richter et al., 2000; Vingerhoets et al., 2001). Second, there is no correspondence between our findings and those of studies examining brain activation as a function of task difficulty (e.g., Barch et al., 1997; D’Esposito et al., 1995). For example, an fMRI study by Barch et al. (1997) found that increasing the difficulty of a working memory task resulted in transient increases in activation of the anterior cingulate, basal ganglia, and regions of the right inferior frontal cortex (areas 44/45/47). In contrast, they found sustained increases in activation of left DLPFC and left inferior frontal cortex under conditions in which retention interval was increased independently of task difficulty. Barch et al. interpreted these findings as evidence for distinct brain areas associated with task difficulty versus working memory, respectively. In the current study, our object-self contrast also revealed activation in left DLPFC and left inferior frontal cortex, which is in line with the latter result. However, one still might argue that increased working memory load in the object task per se contributed to the results. With the exception of the middle occipital gyrus, most of the areas of activation showed greater activation in the object task than in the self task when compared to the control condition. Whether this is truly attributable to differences in working memory or is merely a consequence of the relatively high-level control task we used is a question for future research.

When considered in isolation, the RT and error functions we found for imagined self rotations provide additional insights into the corresponding neural mechanism. Participants were fastest and most accurate for $100^{\circ}$ rotations, which are nearly aligned with one of the major axes of the human body. They were slower and less accurate for rotations of $65^{\circ}$ and $135^{\circ}$, which are oblique to the orthogonal axes of the body. This pattern does not fit the usual monotonic function found in mental rotation studies; however, it is in line with previous RT and error patterns of other imagined self rotation tasks (Wraga et al., 2000, 2004). When we consider these functions with respect to the corresponding pattern of neural activity we found, a partial explanation emerges for the paradox of how imagined self rotations may be constrained by some physical laws pertaining to the body, while at the same time generally defying other physical laws such as traveling without passing through intermediate points of space. The fact that people can mentally travel through space without passing through inter- mediate points may reflect the absence of low-level motor activation. However, the reason why aspects of mental transformations are constrained by other physical laws cannot be understood on the basis of the current findings and warrants further empirical investigation.

In summary, we have demonstrated that different classes of mental rotation are subserved by distinct neural mechanisms. Our imagined object rotation task activated low-level motor areas, whereas our imagined self rotation task did not. These findings emphasize the flexibility of spatial processing mechanisms within the human brain.

\section{Acknowledgements}

This research was supported by Grant NMA202-98-K1166 from the Department of Defense, NIH Grant 5 R01 MH60734, and NSF Grant REC-0106760. We wish to thank Scott Grafton, Tammy LaRoche, and Joe Moran of the Dartmouth Brain Imaging Center for their assistance. We also thank Bill Thompson of Harvard University for helpful comments on an earlier draft of the paper.

\section{References}

Amorim, M., \& Stucchi, N. (1997). Viewer- and object-centered mental explorations of an imagined environment are not equivalent. Cognitive Brain Research, 5, 229-239.

Barch, D. A., Braver, T. S., Nystrom, L. E., Forman, S. D., Nolls, D. C., \& Cohen, J. C. (1997). Dissociating working memory from task difficulty in human prefrontal cortex. Neurospychologia, 35, 1373-1380.

Barnes, J., Howard, R. J., Senior, C., Brammer, M., Bullmore, E. T., Simmons, A., et al. (2000). Cortical activity during rotational and linear transformations. Neuropsychologia, 38, 1148-1156.

Bonda, E., Petrides, M., Frey, S., \& Evans, A. (1995). Neural correlates of mental transformations of the body-in-space. Proceedings of the National Academy of Sciences of the United States of America, 92, 11180-11184.

Brett, M. (2002). The MNI brain and the Talairach atlas. Retrieved May 25, 2003, from Cambridge University Cognition and Brain Sciences Unit Web site: http://www.mrc-cbu.cam.ac.uk/ Imaging/mnispace.html [On-line].

Carpenter, P. A., Just, M. A., Keller, T. A., Eddy, W., \& Thulborn, K. (1999). Graded functional activation in the visuospatial system with the amount of task demand. Journal of Cognitive Neuroscience, 11, 9-24.

Cohen, M. S., Kosslyn, S. M., Breiter, H. C., DiGirolamo, G. J., Thompson, W. L., Anderson, A. K., et al. (1996). Changes in cortical activity during mental rotation: A mapping study using functional magnetic resonance imaging. Brain, 119, 89-100.

Cohen, J., MacWhinney, B., Flatt, M., \& Provost, J. (1993). PsyScope: An interactive graphic system for designing and controlling experiments in the psychology laboratory using Macintosh computers. Behavior Research Methods, Instruments, and Computers, 25, 257-271.

Creem, S. H., Hirsch Downs, T., Wraga, M., Harrington, G. S., Proffitt, D. R., \& Downs, J. H., III. (2001). An fMRI study of imagined self-rotation. Cognitive, Affective, and Behavioral Neuroscience, 1, 239-249.

Creem, S. H., Wraga, M., \& Proffitt, D. R. (2001). Imagining physically impossible transformations: Geometry is more important than gravity. Cognition, 81, 41-64. 
Crozier, S., Sirigu, A., Lehericy, S., van de Moortele, P.-F., Pillon, B., Grafman, J., et al. (1999). Distinct prefrontal activations in processing sequence at the sentence and script level: An fMRI study. Neuropsychologia, 37, 1469-1476.

D’Esposito, M., Detre, J. A., Alsop, D. C., Shin, R. K., Atlas, S., \& Grossman, M. (1995). The neural basis of the central executive system of working memory. Nature, 378, 79-281.

Dupont, P., Orban, G. A., De Bruyn, B., Verbruggen, A., \& Mortelmans, L. (1994). Many areas in the human brain respond to visual motion. Journal of Neurophysiology, 72, 1420-1424.

Easton, R. D., \& Sholl, M. J. (1995). Object-array structure, frames of reference, and retrievalof spatial knowledge. Journal of Experimental Psychology: Learning, Memory, and Cognition, 21, 483-500.

Forman, S. D., Cohen, J. D., Fitzgerald, M., Eddy, W. F., Mintun, M. A., \& Noll, D. C. (1995). Improved assessment of significant activation in functional magnetic resonance imaging (fMRI): Use of a cluster-size threshold. Magnetic Resonance in Medicine, 33, 636-647.

Friston, K. J., Holmes, A. P., Worsley, K. J., Poline, J.-P., Frith, C. D., \& Frackowiak, R. S. J. (1995). Statistical parametric maps in functional imaging: A general linear approach. Human Brain Mapping, 2, 189-210.

Ganis, G., Keenan, J. P., Kosslyn, S. M., \& Pascual-Leone, A. (2000). Transcranial magnetic stimulation of primary motor cortex affects mental rotation. Cerebral Cortex, 10, 175-180.

Harris, I. M., \& Miniussi, C. (2003). Parietal lobe contribution to mental rotation demonstrated with rTMS. Journal of Cognitive Neuroscience, 15, 315-323.

He, S. Q., Dumm, R. P., \& Strick, P. L. (1995). Topographic organization of corticospinal projections from the frontal lobe: Motor areas on the medial surface of the hemisphere. Journal of Neuroscience, 15, 3284-3306.

Howard, I. (1982). Human visual orientation. Chichester: John Wiley.

Huttenlocher, J., \& Presson, C. C. (1997). The coding and transformation of spatial information. Cognitive Psychology, 11, 375-394.

Kosslyn, S. M., DiGirolamo, G. J., Thompson, W. L., \& Alpert, N. M. (1998). Mental rotation of objects versus hands: Neural mechanisms revealed by positron emission tomography. Psychophysiology, $35,151-161$.

Kosslyn, S. M., Thompson, W. L., Wraga, M., \& Alpert, N. M. (2001). Imagining rotation by endogenous versus exogenous forces: Distinct neural mechanisms. NeuroReport, 12, 2519-2525.

Lamm, C., Windischberger, C., Leodolter, U., Moser, E., \& Bauer, H. (2001). Evidence for premotor cortex activity during dynamic visuospatial imagery from single-trial functional magnetic resonance imaging and event-related slow cortical potentials. NeuroImage, 14, 268-283.

Oldfield, R. C. (1971). The assessment and analysis of handedness: The Edinburgh Inventory. Neuropsychologia, 9, 97-113.

Parsons, L. M., Fox, P. T., Downs, J. H., Glass, T., Hirsch, T. B., Martin, C. G., et al. (1995). Use of implicit motor imagery for visual shape discrimination as revealed by PET. Nature, 375, 54-58.

Paus, T., Marrett, S., Worsley, K. J., \& Evans, A. C. (1995). Extraretinal modulation of cerebral blood flow in the human visual cortex: Implications for saccadic suppression. Journal of Neurophysiology, 74, 2179-2183.

Picard, N., \& Strick, P. L. (1996). Motor areas of the medial wall: A review of their location and functional activation. Cerebral Cortex, 9 , $342-353$.

Podzebenko, K., Egan, G. F., \& Watson, J. D. G. (2002). Widespread dorsal stream activation during a parametric mental rotation task, re- vealed with functional magnetic resonance imaging. NeuroImage, 15, 547-558.

Presson, C. C. (1982). Strategies in spatial reasoning. Journal of Experimental Psychology: Learning, Memory, and Cognition, 8, 243-251.

Raichle, M. E. (1998). The neural correlates of consciousness: An analysis of cognitive skill learning. Philosophical Transactions of the Royal Society of London. Series B, Biological Sciences, 353, 1889-1901.

Richter, W., Somorjai, R., Summers, R., Jarmasz, M., Menon, R. S., Gati, J. S., et al. (2000). Motor area activity during mental rotation studied by time-resolved single-trial fMRI. Journal of Cognitive Neuroscience, 12, 310-320.

Rizzolati, G., Camarda, R., Fogassi, L., Gentilucci, M., Luppino, G., \& Matelli, M. (1998). Functional organization of inferior area 6 in the macaque monkey. II. Area F5 and the control of distal movements. Experimental Brain Research, 71, 491-507.

Shelton, A. L., \& McNamara, T. P. (2001). Systems of spatial reference in human memory. Cognitive Psychology, 43, 274-310.

Shepard, R. N., \& Metzler, J. (1971). Mental rotation of three-dimensional objects. Science, 171, 701-703.

Shulman, G. L., Fiez, J. A., Corbetta, M., Buckner, R. L., Miezin, F. M., Raichle, M. E., et al. (1997). Common blood flow changes across visual tasks. II. Decreases in cerebral cortex. Journal of Cognitive Neuroscience, 9, 648-663.

Snyder, L. H., Batista, A. P., \& Andersen, R. A. (2000). Intention-related activity in the posterior parietal cortex: A review. Vision Research, 40, 1433-1441.

Tagaris, G. A., Kim, S.-G., Strupp, J. P., Andersen, P., Ugurbil, K., \& Georgopoulos, A. P. (1997). Mental rotation studied by functional magnetic resonance imaging at high field (4 Tesla): Performance and cortical activation. Journal of Cognitive Neuroscience, 9, 429-432.

Talairach, J., \& Tournoux, P. (1988). Co-planar stereotaxic atlas of human brain. New York: Georg Thieme Verlag.

Vingerhoets, G., Santens, P., Van Laere, K., Lahorte, P., Dierckx, R. A., \& De Reuck, J. (2001). Regional brain activity during different paradigms of mental rotation in healthy volunteers: A positron emission tomography study. Neurolmage, 13, 381-391.

Wexler, M., Kosslyn, S. M., \& Berthoz, A. (1998). Motor processes in mental rotation. Cognition, 68, 77-94.

Wraga, M. (2003). Thinking outside the body: An advantage for spatial updating during imagined versus physical self-rotation. Journal of Experimental Psychology: Learning, Memory, and Cognition, 29, 993-1005.

Wraga, M., Creem, S. H., \& Proffitt, D. R. (2000). Updating displays after imagined object and viewer rotations. Journal of Experimental Psychology: Learning, Memory, and Cognition, 26, 151-168.

Wraga, M., Creem-Regehr, S. H., \& Proffitt, D. R. (2004). Spatial updating of virtual displays during self- and display rotation. Memory \& Cognition, 32, 399-415.

Wraga, M., Thompson, W. T., Alpert, N. M., \& Kosslyn, S. M. (2003). Implicit transfer of motor strategies in mental rotation. Brain and Cognition, 52, 135-143.

Zacks, J. M., Ollinger, J. M., Sheridan, M. A., \& Tversky, B. (2002). A parametric study of mental spatial transformations of bodies. $\mathrm{Neu}$ roImage, 16, 857-872.

Zacks, J., Rypma, B., Gabrieli, J., Tversky, B., \& Glover, G. (1999). Imagined transformations of bodies: An fMRI study. Neuropsychologia, 37, 1029-1040.

Zacks, J. M., Vettel, J. M., \& Michelon, P. (2003). Imagined viewer and object rotations dissociated with event-related fMRI. Journal of Cognitive Neuroscience, 15, 1002-1018. 